\title{
A THEOREM ON ONE-TO-ONE MAPPINGS ONTO THE PLANE
}

\author{
R. F. DICKMAN, JR.
}

Let $X$ be a generalized continuum, i.e. let $X$ be a connected, locally compact, separable metric space, and let $F$ be a one-to-one continuous map of $X$ onto the plane. A point $x \in X$ will be called a cut-point (respectively, a local separating point) of $X$ provided $x$ separates $X$ (respectively, $x$ separates some open set in $X$ ). See [7, pp. 41, 61]. By an $m$-manifold, $m \geqq 0$, we shall mean a separable metric space such that every point has a neighborhood homeomorphic to an open subset of a closed $m$-cell. All terms not defined here may be found in [7]. There are several known conditions on $X$ that imply that $F$ is a homeomorphism. In [4] L. C. Glaser proved that if $X$ is a 2-manifold that satisfies several strong conditions, then $F$ is a homeomorphism. In [2] E. Duda proved that if $X$ is a locally connected generalized continuum that has the property that the complement of any compact set has exactly one component with a noncompact closure, then $F$ is a homeomorphism. In [1] the author used Duda's result to show that if $X$ is the product of two noncompact, locally connected, generalized continua, then $F$ is a homeomorphism. In [5] D. H. Pettey was able to remove the conditions imposed by Glaser and prove that if $X$ is any 2-manifold, $F$ is a homeomorphism. There are several examples of one-to-one continuous maps of 3-manifolds on to $E^{3}$ which are not homeomorphisms. See [6] or Lemma 2 of [3]. It is an open question as to whether every one-to-one continuous map of a locally connected generalized continuum onto the plane is a homeomorphism. The purpose of this note is to prove the following:

THEOREM. If $X$ is a locally connected generalized continuum without any local separating points and $F$ is a one-to-one continuous map of $X$ onto the plane, then $X$ is a 2-manifold and $F$ is a homeomorphism.

Proof. Let $x \in X$. Since $x$ does not separate any open set in $X, x$ does not separate $X$ and by Proposition (4.15) of $[7$, p. 50] there exists a connected open set $V$ of $X$ containing $x$ such that $\bar{V}$ is compact and $X-V$ is connected. By [7, Proposition (15.43), p. 22] there exists a connected open set $W$ containing $x$ such that $\bar{W} \subset V$ and $\bar{W}$ is locally connected. Furthermore, since no point of $W$ separates $W$, $\bar{W}$ has no cut-points. Since $F(\bar{W})$ is a subset of $F(V)$ and since

Received by the editors March 8. 1968. 
$F(X-V)$ is an unbounded connected set, $F(X-V)$ lies entirely in the unbounded complementary domain of $F(\bar{W})$. Thus if $H$ denotes the union of $F(\bar{W})$ and all of the bounded complementary domains of $F(\bar{W}), H$ is a closed, compact and connected subset of $F(V)$ that contains the closed 2-cell bounded by any simple closed curve in $H$. Every complementary domain of $F(\bar{W})$ has no cut-points and $F(\bar{W})$ has no cut-points, and since the boundary of every complementary domain of $F(\bar{W})$ is a nondegenerate subset of $F(\bar{W}), H$ has no cutpoints. Since $F(\bar{W})$ is locally connected, it follows from [7, Theorem (4.4), p. 113] that $H$ is locally connected. Thus $H$ is a compact, connected, locally connected subset of the plane that has no cut-points and contains the closed 2-cell bounded by any simple closed curve in $H$; and hence, by the characterization of the closed 2-cell in [2], $H$ is a closed 2-cell. Since $F \mid \bar{V}$ is a homeomorphism and since $H \subset V$, $B=F^{-1}(\bar{H})$ is a closed 2-cell in $X$ and $x$ is interior to $B$. Hence $X$ is a 2-manifold, and by Theorem 2 of [5], $F$ is a homeomorphism.

\section{REFERENCES}

1. R. F. Dickman, Jr., Compactness of mappings on products of locally connected generalized continua, Proc. Amer. Math. Soc. 18 (1967), 1093-1094.

2. Edwin Duda, $A$ theorm on one-to-one mappings, Pacific J. Math. 19 (1966), 253-257.

3. L. C. Glaser, Dimension lowering monotone non-compact mappings of $E^{n}$, Fund. Math. 58 (1966), 177-181.

4. - 1-1 continuous mappings onto $E^{n}$, Amer. J. Math. 88 (1966), 237-243.

5. D. H. Pettey, One-to-one continuous mappings into $E^{2}$, Notices Amer. Math. Soc. 15 (1968), 165.

6. K. Whyburn, $A$ non-topological 1-1 mapping onto $E^{3}$, Bull. Amer. Math. Soc. 71 (1965), 533-537.

7. G. T. Whyburn, Analytic topology, Amer Math. Soc. Colloq. Publ., Vol. 28, Amer. Math. Soc., Providence, R. I., 1942.

The University of Miami 